\title{
Neonatal Mononuclear Cell Metabolism: Further Evidence For Diminished Monocyte Function in the Neonate
}

\author{
MAYA DAS, THEODORE HENDERSON, AND STEPHEN A. FEIG \\ The Gwynne Hazen Cherry Memorial Laboratories, Department of Pediatrics, Division of Hematology/Oncology, \\ UCLA School of Medicine, Los Angeles, California, USA
}

\begin{abstract}
Summary
The metabolic capacity of neonatal monocytes was compared to the metabolic capacity of adult monocytes by two entirely different methods: the selective diminution of monocyte contamination of whole mononuclear cells and the isolation of relatively purified populations of monocytes.

Monocyte removal from whole mononuclear cells produced a diminution in the pyruvate kinase (PK) activity (from $28.6 \pm 1.1$ to $15.6 \pm 1.2 \mathrm{nmoles} / \mathrm{min} / 10^{7}$ cells) and an increase in adenosine triphosphate (ATP) content (from $7.9 \pm 1.0$ to $9.5 \pm 0.8$ nmoles/ $10^{7}$ cells) in adult cells. No change in PK activity (from $13.5 \pm 1.3$ to $14.0 \pm 1.3$ ) was observed in cord cells, but the ATP content of cord cells was higher after monocyte depletion (from $4.7 \pm 0.5$ to $6.2 \pm 0.7$ ).

The suggestion of metabolic vulnerability was confirmed by metabolic analysis of isolated adult and cord monocytes. The PK activity of adult monocytes was greater than that of cord monocytes ( $57 \pm 9$ and $25 \pm 0.3$, respectively) and the ATP content of adult monocytes $(5.7 \pm 0.2)$ was greater than that of cord monocytes $(2.3 \pm 0.1)$.

The data confirm prior observations of diminished energy metabolism in neonatal mononuclear cells and suggest that the metabolic perturbations may, in part, correlate with functional immaturity of the neonatal monocyte.
\end{abstract}

\section{Speculation}

Monocytes have a glycolytic rate that far exceeds that of other mononuclear cells. The presence of an increased rate of energy synthesis, however, is not accompanied by an increase in ATP content. The metabolic requirements of monocytes are, therefore, greater than for other mononuclear cells. This suggests that monocytes may be susceptible to dysfunction secondary to metabolic stress (increased metabolic requirements or diminished metabolic capacity). The neonatal monocyte appears to be much more metabolically vulnerable than the adult monocyte and this may, in part, account for the immune dysfunction observed in the newborn infant.

Observations of increased frequency and severity of infections among newborn infants suggest that the immature immune system may not function as well as that of older individuals. A variety of data, reviewed previously (5), suggests that the cell-mediated immune function of newborns is impaired compared to that of older children and adults, but the specific nature of this dysfunction is unclear.

In an attempt to clarify the nature and cause of neonatal immune dysfunction, we began a study of neonatal mononuclear cell metabolism. We demonstrated diminished content of several enzymes and ATP in the mononuclear cells of newborn infants
(5). In particular, we noted decreased activity of PK (ATP: pyruvate phosphotransferase-EC: 2.7.1.40) activity, which increased during the first year of life to the level found in adult mononuclear cells. Furthermore, the PK activity of adult cells could be stimulated by exposure to high concentrations of phytohemagglutinin, while neonatal PK activity was unaffected by phytohemagglutinin. Glycolytic metabolism of neonatal mononuclear cells was reduced by $10-20 \%$ compared to adult cells, but these differences were not statistically significant, because of the variability of metabolism between subjects.

The data suggested diminished metabolic capacity of neonatal mononuclear cells. No specific correlation of the metabolic perturbations could be defined because the cell populations we studied were so heterogeneous. The availability of new techniques for separation of mononuclear cell subtypes has allowed us to pursue this observation $(1,12)$.

We postulated that diminished metabolism of the neonatal monocyte might be responsible for some of the observed differences between neonatal and adult mononuclear cells. We recognize that preparative methods might affect cell function and metabolism. Therefore, monocyte metabolism was assessed in two ways. First, mononuclear cells were studied before and after monocyte removal. Second, monocytes were isolated by glass adherence and then harvested for metabolic study in a relatively pure suspension. The data strongly suggest that the neonatal monocyte is deficient in PK activity and ATP content compared to the adult monocyte. The functional significance of this observation remains to be defined.

\section{MATERIALS AND METHODS}

Blood specimens were obtained from adults and from the placental end of the cut umbilical cord after obtaining informed consent. Mononuclear cells were separated on Ficoll-Isopaque gradient (3). An aliquot of mononuclear cells was removed for study and the remainder were exposed to a suspension of iron filings (Lymphocyte Separator Reagent, Technicon Instruments Corp., Tarrytown, NY 10591). After incubation for $30 \mathrm{~min}$ at $37^{\circ}$, the cells were centrifuged again over a Ficoll-Isopaque gradient and the enriched lymphocytes were harvested from the interface (14).

Alternatively, mononuclear cells were suspended in RPMI medium 1640 with $20 \%$ autologous serum, placed in $25 \mathrm{ml}$ Falcon flasks, and centrifuged for $5 \mathrm{~min}$ at $100 \times \mathrm{g}$. After $30 \mathrm{~min}$ incubation at room temperature, nonadherent cells were removed by washing vigorously, four times, with warm medium. Adherent cells were then removed by incubation in Roswell Park Memorial Institute medium 1640 with $20 \%$ autologous serum and $0.4 \%$ lidocaine for $30 \mathrm{~min}$. The cells were then washed three times in a buffered physiologic saline with $1 \%$ human albumin and glucose (5). 
Table 1. Effect of monocyte removal on PK activity and ATP content of adult and neonatal mononuclear cells ${ }^{1}$

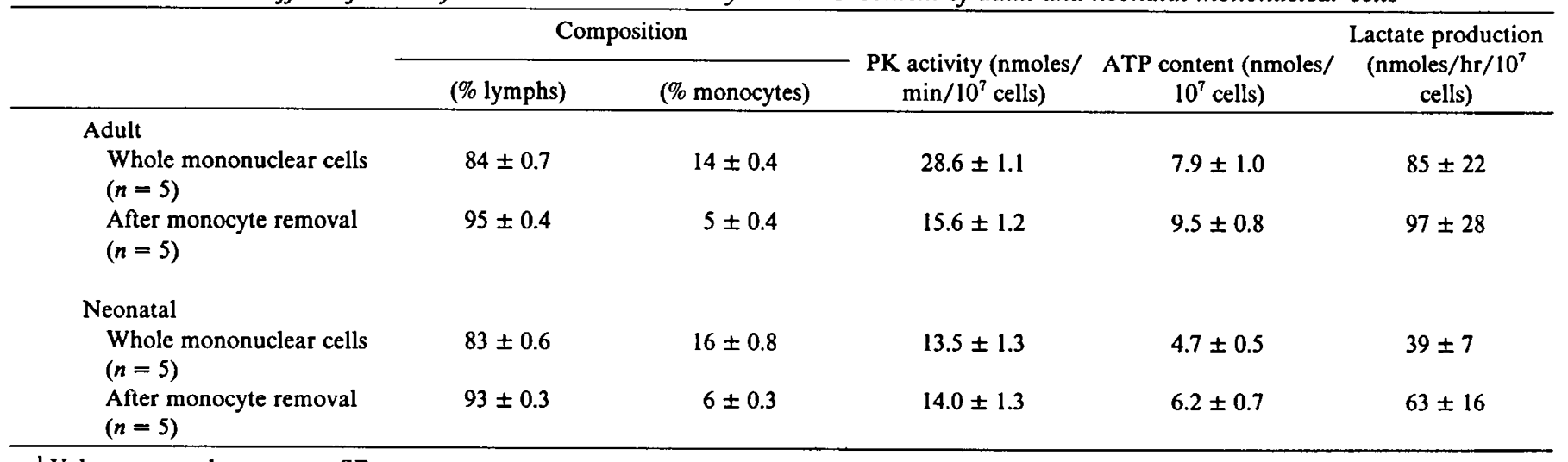

' Value expressed as mean $\pm S E$.

Table 2. Enzyme activity and ATP content of adult and neonatal monocytes $^{1,2}$

\begin{tabular}{|c|c|c|c|c|}
\hline & \multicolumn{2}{|c|}{ Composition } & \multirow{2}{*}{$\begin{array}{c}\text { PK activity } \\
\text { (nmoles/ } \\
\text { min } / 10^{7} \text { cells) }\end{array}$} & \multirow{2}{*}{$\begin{array}{c}\text { ATP content } \\
\text { (nmoles } / 10^{7} \\
\text { cells) }\end{array}$} \\
\hline & $\begin{array}{c}\text { (\% mono- } \\
\text { cytes) }\end{array}$ & (\% lymphs) & & \\
\hline $\begin{array}{l}\text { Adult } \\
\quad(n=8) \\
\text { Neonatal }\end{array}$ & $95 \pm 1$ & $5 \pm 1$ & $57 \pm 9$ & $5.7 \pm 0.2$ \\
\hline$(n=10)$ & $96 \pm 0.3$ & $4 \pm 0.3$ & $25 \pm 0.3$ & $2.3 \pm 0.1$ \\
\hline
\end{tabular}

Cells were counted manually. Differential counts were performed on cytocentrifuge preparations stained with Wright and Esterase stains. Even on cord blood specimens, nucleated red blood cell contamination was insignificant.

Metabolic studies were performed as previously described (5).

\section{RESULTS}

The compositions of the cell populations studied were similar when adult and cord samples were compared (Table 1). Whole mononuclear cells were greater than $80 \%$ lymphoid and approximately $15 \%$ monocytic. Exposure to iron filings and subsequent centrifugation reduced the monocyte content of the samples to approximately $5 \%$.

The PK activity of adult mononuclear cells was markedly reduced by the removal of monocytes $(P<0.001)$. In contrast, the PK activity of cord cells was unaffected (Table 1). As previously reported, the ATP content of adult mononuclear cells exceeded that of cord mononuclear cells (5). Monocyte removal seemed to be associated with a slight increase in the ATP content of the remaining cells in both cord and adult cells (Table 1). This observation suggests that the ATP content of monocytes may be lower than that of other mononuclear cells.

The composition of isolated monocyte populations was similar in adult and cord samples (Table 2). The PK activity of isolated monocytes was greater than that for monocyte-depleted mononuclear cells in both adult and cord specimens (Tables 1 and 2). While the PK activity of monocyte-depleted mononuclear cells was similar in cord and adult samples (Table 1), adult monocyte PK activity was greater than cord monocyte PK activity (Table 2). Cord monocyte ATP content was markedly reduced compared to the ATP content of adult monocytes (Table 2).

Lactate production of adult mononuclear cells appeared slightly greater than that of cord mononuclear cells (Table 1). Lactate production of enriched lymphocytes appeared slightly greater than that of whole mononuclear cells. These differences were not statistically significant and the findings were consistent with our previous report (5).

We were surprised to observe increased lactate production in the lymphocyte enriched population of neonatal and adult mononuclear cells, because monocytes are well known to have a metabolic rate much greater than that of lymphocytes $(4,13)$. Thus, monocyte removal was expected to diminish the metabolic activity of residual mononuclear cells. We suspect that the small numbers of contaminating monocytes in the enriched lymphocyte samples may have been subjected to metabolic stimulation by the ingestion of iron. Unfortunately, the size of the cord blood samples did not permit the isolation of neonatal monocytes in sufficient quantity to measure lactate production.

\section{DISCUSSION}

The nature of the neonatal immune defect is not clear. In fact, it seems likely that the dysfunction is complex and may involve partial deficits in several aspects of the immune reponse. These studies focus upon the metabolic differences between neonatal and adult monocytes.

Klein et al. (6) have described an impairment of neonatal monocyte chemotaxis. Their observation, using a new method, is at variance with prior and subsequent descriptions of normal monocyte chemotaxis $(8,11)$. The differences between the conclusions of the groups may reflect methodologic differences, particularly in the nature of the chemotactant, and the medium in which the experiments were carried out. Assessments of neonatal monocyte bactericidal activity $(8,10)$ and nitroblue terazolium reduction (7) show functional capability similar to that of adult cells.

The studies reported here do not reflect the metabolic requirements of any particular cellular function. Glycolysis is the primary pathway of energy synthesis in human mononuclear cells $(4,13)$. PK is a critical enzyme in the control of glycolysis and the step at which half the ATP synthesis of glycolysis takes place. Thus, the combined measurements of PK activity and ATP content represent an assessment of a major portion of energy turnover capacity of all mononuclear cells.

It is clear that methods of cell preparation may influence the subsequent metabolic and functional activities of isolated cell populations. For that reason, cells were prepared by two reciprocal methods. First, the contribution of monocytes to total mononuclear cell metabolism was assessed by the removal of monocytes from the original population. Second, monocytes were isolated for metabolic study. Both methods demonstrated relative deficiency of PK activity and ATP content in neonatal monocytes compared to adult monocytes. It is possible that the observations we have made might be the result of artifacts of our preparative methods. However, the selective effect on neonatal cells observed by two entirely different approaches is indicative of a basic difference in the metabolic capacity or susceptibility of neonatal monocytes compared to adult monocytes. 
Recent data would suggest that circulating monocytes may be subclassified into subsets by size $(1,9)$ and by the presence of $\mathrm{Fc}$ receptors on their membranes (2). It seems likely that monocytes may represent an extremely heterogeneous group of cells. We suspect that the reduced PK activity and ATP content is representative of metabolic capacity of neonatal monocytes. Alternatively, the observations may represent selection of different subpopulations in neonatal and adult populations of mononuclear cells. We cannot eliminate this latter possibility because we have no data regarding the heterogeneity of monocyte subsets and their relative enrichment in the populations of cells tested.

Although monocytes vary according to size, approximately half are the size of lymphocytes and half are 50-70\% larger $(1,9)$. Thus, level of monocyte ATP, expressed in this report on a per cell basis, would reflect an even lower concentration if expressed on a molar basis (i.e., per liter of cell water).

The present data suggests that monocytes have a high level of metabolic activity, but maintain a relatively low level of ATP. In that perspective, monocytes appear to be particularly vulnerable to metabolic stress. The observations we have made suggest that neonatal monocytes may be even more susceptible to metabolic depletion. This is consistent with the hypothesis that monocyte dysfunction secondary to metabolic vulnerability, may play a role in the immunologic immaturity of the newborn infant. Further studies are necessary to evaluate this hypothesis.

\section{REFERENCES AND NOTES}

1. Arenson, E. B., and Seeger, R. C.: Cell surface differentiation antigens of human mononuclear phagocytes. Clin. Res., 26: 183A (1978).
2. Barrett, S. G.: Differences in immune receptors on human blood mononuclear and neutrophilic phagocytes. Clin. Res., 26: 340A (1978).

3. Böyum, A.: A one stage procedure for isolation of granulocytes and lymphocytes from human blood. Scand. J. Clin. Lab.Invest., 21: 51 (1968).

4. Cline, M. J., and Lehrer, R. I.: Phagocytosis by human monocytes. Blood, 32: 423 (1968).

5. Das, M., Klein, W., and Feig, S. A.: Glycolytic metabolism of neonatal mononuclear cells. Pediatr. Res., 11: 1026 (1977).

6. Klein, R. B., Fischer, T. J., Gard, S. E., Biberstein, M., Rich, K. C., and Stiehm, E. R.: Decreased mononuclear and polymorphonuclear chemotaxis in human newborns, infants, and young children. Pediatrics, 60: 467 (1977).

7. Kretschmer, R. R., Papierniak, C. K., Stewardson-Krieger, P., Bamzai, A. K., and Gotoff, S. P.: Quantitative nitrobluetetrazolium reduction by normal newborn monocytes. J. Pediatr., 90: 306 (1977).

8. Kretschmer, R. R., Stewardson, P. B., Papierniak, C. K., and Gotoff, S. P.: Chemotactic and bactericidal capacities of human newborn monocytes. J. Immunol., 117: 1303 (1976).

9. Morris, R. J., Norris, D. A., and Sanderson, R. J.: Monocytes in ADCC: evidence for two functional subtypes. Clin. Res., 26: 123A (1978).

10. Orlowski, J. P., Sieger, L., and Anthony, B. F.: Bactericidal capacity of monocytes of newborn infants. J. Pediatr., 89: 797 (1976).

11. Pahwa, S. G., Pahwa, R., Grimes, E., and Smithwick, E.: Cellular and humoral components of monocyte and neutrophil chemotaxis in cord blood. Pediatr. Res., 11: 677 (1977).

12. Rabinovitch, M., and DeStefano, M. J.: Use of the local anesthetic lidocaine for cell harvesting and subcultivation. In vitro, 11: 379 (1975).

13. Vannotti, A.: Metabolic pattern of leukocytes within the circulation and outside it. Ciba Found. Study Group, 10: 79 (1961).

14. Zucker-Franklin, D.: The percentage of monocytes among mononuclear cell fractions obtained from normal human blood. J.Immunol., 112: 234 (1974).

15. This research was supported by a research support grant from the National Institutes of Health (RR-5354) and the Karen Brigham Memorial Fund.

16. Requests for reprints should be addressed to: Stephen A. Feig, M.D., Associate Professor, Department of Pediatrics, UCLA School of Medicine, 10833 Le Conte, Los Angeles, CA 90024 (USA).

17. Received for publication July $25,1977$.

18. Accepted for publication June 8, 1978. 